\title{
The recent secular trend in grip strength among older adults: findings from the English Longitudinal Study of Ageing
}

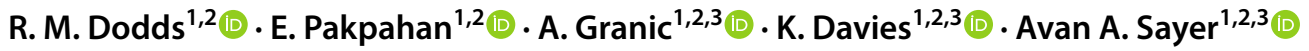

Received: 22 November 2018 / Accepted: 16 February 2019 / Published online: 6 March 2019

(c) The Author(s) 2019

\section{Key summary points}

Aim Our aim was to investigate whether there has been a recent secular trend in the grip strength of older English adults, using data from the English Longitudinal Study of Ageing (ELSA).

Findings We found evidence of a slight decline in mean grip strength between 2004 and 2013. This decline is equivalent to 65-year-olds' mean strength declining to that previously seen in individuals at age 69, and did not appear to be explained by differences in lifestyle risk factors.

Message These findings are important since they raise the possibility that more recent cohorts of older people remain at similar, or possibly slightly greater, risk of the adverse consequences of weak muscle strength.

\begin{abstract}
Purpose Weaker grip strength in older adults is associated with adverse health outcomes and is a key component of sarcopenia. The secular trend of grip strength is, therefore, relevant in the setting of ageing populations. A recent study suggested differences in this trend among countries in mainland Europe. We used data from the English Longitudinal Study of Ageing (ELSA) to investigate the recent secular trend of older English adults.

Methods We used data on participants aged 50-89 having their first measurement of grip strength in waves 2 (2002/2003), $4(2008 / 2009)$ or $6(2012 / 2013)$ of ELSA. Grip was measured using a Smedley dynamometer. We expressed grip values as $Z$-scores (number of standard deviations above the age and gender mean from normative data) for use in linear regression analyses examining the annual secular trend after adjustment for potential confounders.

Results We included a total of 11,476 participants from the three waves of ELSA. Grip strength declined across the three waves, with mean (SD) Z-scores of $0.01(0.94),-0.06(0.97)$ and $-0.20(0.98)$ in waves 2,4 and 6 , respectively. The annual $Z$-score decline after adjustments was 0.03 SDs (95\% CI 0.02, 0.03) per year.

Conclusion We saw evidence of a recent slight decline in the grip strength of older English adults. Over the 9-year period of this study, the decline seen is equivalent to 65-year-olds' mean strength declining to that previously seen in individuals at age 69. Further monitoring of secular trends in grip strength and investigation of possible causes are warranted.
\end{abstract}

Keywords Grip strength $\cdot$ Older people $\cdot$ Secular trend $\cdot$ Sarcopenia

R. M. Dodds

richard.dodds@newcastle.ac.uk

1 AGE Research Group, Institute of Neuroscience, Faculty of Medical Sciences, Newcastle University, Newcastle upon Tyne, UK

2 NIHR Newcastle Biomedical Research Centre, Newcastle upon Tyne Hospitals NHS Foundation Trust and Faculty of Medical Sciences, Newcastle University, Newcastle upon Tyne, UK

3 Newcastle University Institute for Ageing, Newcastle upon Tyne, UK 


\section{Introduction}

Weaker grip strength is associated with disability [1, 2], morbidity [3] and mortality [4] and is a key component of the ageing syndrome of sarcopenia in the revised European consensus on definition and diagnosis [5]. Studies conducted during the twentieth century in high-income countries have typically shown a secular increase in the grip strength of children and adolescents [6] as well as a slight increase in the peak grip strength achieved in early adulthood [7]. However, there has been less exploration of the secular trend in the grip strength of older adults; if present, such trends could provide useful information for the planning of health and social care services.

There is recent evidence of a decline in self-reported physical functioning among older Germans between 2006 and 2012; this was in contrast to an improvement in cognitive functioning over the same period [8]. The grip strength of older people in Germany has also shown a slight decline over a similar period, as has that in the Netherlands and Belgium [9]. This is contrast to an apparent secular increase in grip strength in Denmark, Sweden, Italy and Spain seen in the same study [9].

To our knowledge there has not been an assessment made of whether there has been a recent secular trend in grip strength among older people in England. We, therefore, used data from the English Longitudinal Study of Ageing (ELSA) to investigate this question.

\section{Methods}

We used data from ELSA, a broadly representative sample of adults aged 50+ drawn from the Health Survey for England and first assessed in 2002/03 and then every 2 years since including refreshment samples at waves 3,4 and 6 [10, 11]. Partners of study members were also eligible to participate. The study has performed health assessments including grip strength in alternate waves (every 4 years) beginning in 2004. Ethical approval for all ELSA waves was obtained from NHS Research Ethics Committees under the National Research and Ethics Service.

\section{Participant characteristics}

We considered a priori factors with the potential to confound a secular trend in grip strength. The occupational socioeconomic position of each participant (or that of their partner, if higher) has been assessed in ELSA using the National Statistics Socio-economic Classification (NS-SEC) and divided into routine and manual, intermediate and managerial and professional [12]. Cigarette smoking was grouped into current smoker, previous smoker or never smoked. The self-reported frequency of different physical activities was used to classify participants into those who were inactive and those who undertook light, moderate or vigorous activities at least once a week [13]. Finally, measured height and weight were used to calculate body mass index, categorised as $<20, \geq 20$ and $<25, \geq 25$ and $<30$ and $\geq 30 \mathrm{~kg} / \mathrm{m}^{-2}$.

\section{Measurement of grip strength and comparison with British normative data}

We used grip strength data from waves 2, 4 and 6 of ELSA, collected in 2004-2005, 2008-2009, and 2012-2013, respectively. At each of these waves, participants were consented to having their grip strength measured. Three measurements of grip strength were attempted using both hands using a Smedley dynamometer and we used the maximum of the available values in analyses [14]. We used data from only the first time point in which grip strength was measured for each participant, to avoid practice effects. The ages of participants aged 90 and over are collapsed into a single category in the ELSA data for confidentiality reasons. We, therefore, excluded those aged $90+$ at first grip strength measurement. We also excluded the small number of participants (all partners) aged under 50 at the time of their first grip strength measurement.

We wished to be able to combine grip strength measurements that had been taken at different ages in both men and women. We, therefore, used our previous British normative data for grip strength [15] to express each participant's reading into a $Z$-score. Each $Z$-score is calculated as the participant's grip strength value less the mean expected for their age and gender, divided by the grip strength standard deviation (SD) for their age and gender. As such Z-scores have no units $(\mathrm{kg} / \mathrm{kg})$. $Z$-scores of +1 and -1 indicate grip strength values one standard deviation above and below, respectively, that expected for age and gender.

\section{Statistical analyses}

We calculated descriptive statistics and used the $t$ test (unpaired and equal variance) to examine changes in grip strength by wave within each 10-year age-group and gender. The ELSA dataset includes the month and year of each grip strength measurement, which we expressed as the number of years (including a decimal component for the number of months) since the start of the wave 2 fieldwork. We expressed grip strength values as $Z$-scores (as described above). We performed linear regression models using the date of grip strength measurement as the exposure and grip strength $Z$-score as the outcome. We repeated the linear regression models with adjustment for the potential 


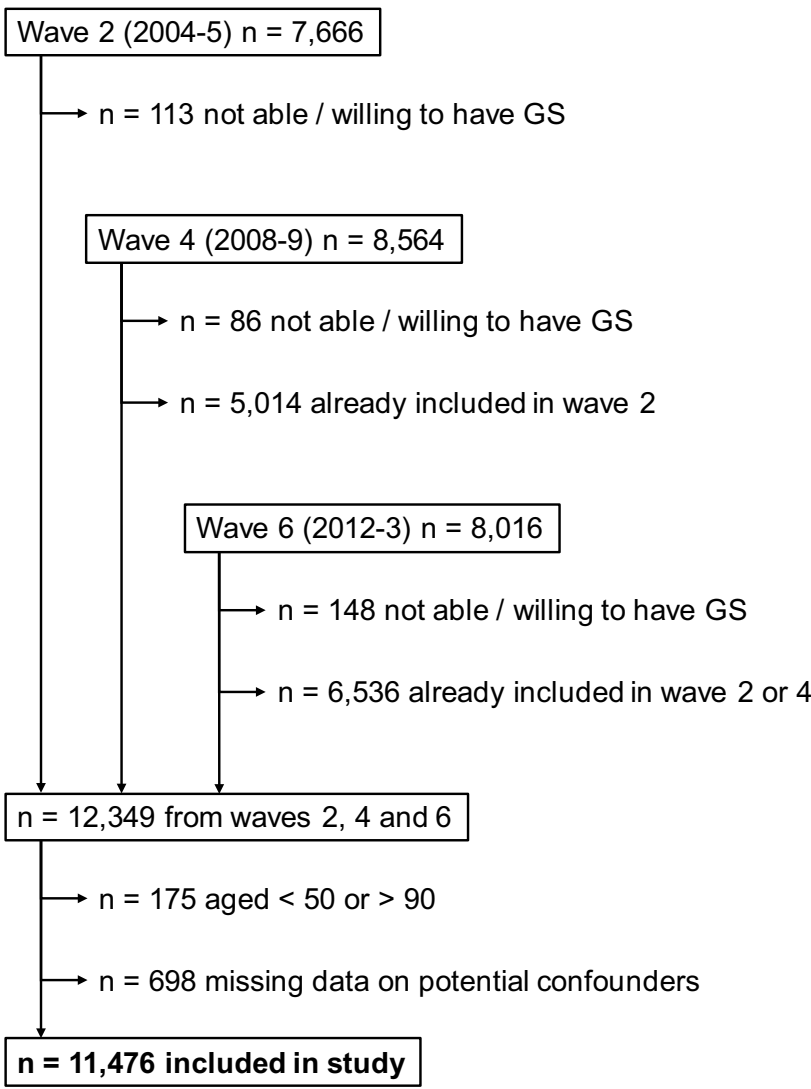

Fig. 1 Flowchart of study participants. Numbers participating in the health assessment at waves 2, 4 and 6 shown. GS grip strength

confounders shown above. We included quadratic terms in the model and tested for evidence of a departure from a linear secular trend in grip strength. We also tested for evidence of interactions by gender and by age group. We performed all analyses using Stata version 14.0 [16].

\section{Results}

A total of 11,476 participants had grip strength measured for the first time in the study in the waves spanning 2004-2013 and complete information on potential confounders, as shown in Fig. 1. In comparison with participants included from wave 2, those in waves 4 and 6 were more likely to have never smoked, to undertake higher levels of physical activity and those in wave 6 were more likely to be of managerial occupational social class, as shown in Table 1. Those in later waves were also younger on average, with mean ages of 66, 61 and 58 years in waves 2,4 and 6 , respectively.

As expected, mean grip strength decreased with age and was higher in men than women in all three waves. There was a trend towards lower mean grip strength in later study waves within most of the gender and age strata, as shown in
Fig. 2. An example is the comparison of mean grip strength in men aged 50-59 in wave 2 of $46.4 \mathrm{~kg}$ (95\% CI 45.9, 46.9) and those in the same age-range in wave 6 of $44.8 \mathrm{~kg}(95 \%$ CI 43.9, 45.7). The mean grip strength $Z$-scores for men and women combined also showed a decline in grip strength between waves, with mean (SD) values of $0.01(0.94)$ in wave $2,-0.06(0.97)$ in wave 4 and $-0.20(0.98)$ in wave 6 .

The linear regression model for grip strength $Z$-scores using the date of measurement as the only predictor showed a 0.02 SDs $(95 \%$ CI $0.02,0.03)$ decrease in grip strength per year of the study. As expected, BMI below 20, current smoking, lower physical activity and routine occupational social class were all associated with lower grip strength. The decrease in grip strength $Z$-score per year became more marked when adjusted for these potential confounders: 0.03 SDs (95\% CI $0.02,0.03)$ per year (Table 2). In sensitivity analyses there was no evidence of interactions between date of measurement and gender or age group.

\section{Discussion}

\section{Summary of findings}

We investigated the secular trend in grip strength between 2004 and 2013 in those participants aged 50-89 using data from ELSA, an English ageing cohort study. We found evidence of a slight decline in mean grip strength across this period. This decline did not appear to be explained by differences in lifestyle risk factors-rather, those in more recent waves had lower levels of factors that were associated with weaker grip strength and hence the decline was more marked after adjustments. These findings are important since they raise the possibility that more recent cohorts of older people remain at similar, or possibly slightly greater, risk of the adverse consequences of weak muscle strength.

\section{Interpretation of findings}

We saw a small decline in grip strength $Z$-score of 0.03 SDs per year after adjustment. It is possible to illustrate this decline by applying it to the mean (SD) grip strength values at age 65 from our British normative data for women of $25.3 \mathrm{~kg} \mathrm{(6.0)}$ and that of men of $42.3 \mathrm{~kg}$ (8.6) [15]. Over 9 years the decline from our linear regression model would result in a reduction of mean grip strength $1.6 \mathrm{~kg}$ or $6 \%$ in women and $2.3 \mathrm{~kg}$ or $5 \%$ in men. Alternatively, such a population decline at age 65 would be equivalent to women and men having average strength previously seen at age 69, i.e., when 4 years older.

This decline may be artefactual, for example due to a bias towards those with weaker grip strength in later recruits to ELSA. The measured confounders in this study suggest 
Table 1 Characteristics of included participants by wave

\begin{tabular}{|c|c|c|c|c|c|}
\hline \multirow{2}{*}{$\begin{array}{l}\text { Characteristic (all } n \\
\text { (\%) unless shown) }\end{array}$} & \multirow{2}{*}{$\begin{array}{l}\text { Wave } 2(2004-2005) \\
(n=7018)\end{array}$} & \multicolumn{2}{|c|}{ Wave 4 (2008-2009) } & \multicolumn{2}{|c|}{ Wave 6 (2012-2013) } \\
\hline & & $(n=3206)$ & $P^{*}$ & $(n=1252)$ & $P^{*}$ \\
\hline Age [mean (SD)] & $66.0(9.1)$ & $60.9(7.9)$ & $<0.01$ & $58.3(7.9)$ & $<0.01$ \\
\hline Female gender & $3812(54.3 \%)$ & $1710(53.3 \%)$ & 0.4 & $682(54.5 \%)$ & 0.9 \\
\hline Occupational SEP & & & 0.7 & & 0.01 \\
\hline Routine & $2246(32.0 \%)$ & $867(27.0 \%)$ & & $374(29.9 \%)$ & \\
\hline Intermediate & $1754(25.0 \%)$ & $735(22.9 \%)$ & & $284(22.7 \%)$ & \\
\hline Managerial & $3018(43.0 \%)$ & $1604(50.0 \%)$ & & $594(47.4 \%)$ & \\
\hline BMI [mean (SD)] & $27.9(4.8)$ & $28.2(5.2)$ & 0.01 & $28.3(5.3)$ & $<0.01$ \\
\hline BMI categories & & & 0.07 & & $<0.01$ \\
\hline$<20$ & $184(2.6 \%)$ & $81(2.5 \%)$ & & $37(3.0 \%)$ & \\
\hline$\geq 20$ and $<25$ & $1746(24.9 \%)$ & $813(25.4 \%)$ & & $322(25.7 \%)$ & \\
\hline$\geq 25$ and $<30$ & $3055(43.5 \%)$ & $1314(41.0 \%)$ & & $479(38.3 \%)$ & \\
\hline$\geq 30$ & $2033(29.0 \%)$ & $998(31.1 \%)$ & & $414(33.1 \%)$ & \\
\hline Smoking & & & $<0.01$ & & $<0.01$ \\
\hline Current & $1012(14.4 \%)$ & $513(16.0 \%)$ & & $241(19.2 \%)$ & \\
\hline Previous & $3416(48.7 \%)$ & $1337(41.7 \%)$ & & $495(39.5 \%)$ & \\
\hline Never & $2590(36.9 \%)$ & $1356(42.3 \%)$ & & $516(41.2 \%)$ & \\
\hline Physical activity & & & $<0.01$ & & $<0.01$ \\
\hline Inactive & $433(6.2 \%)$ & $230(7.2 \%)$ & & $83(6.6 \%)$ & \\
\hline Mild & $1033(14.7 \%)$ & $380(11.9 \%)$ & & $163(13.0 \%)$ & \\
\hline Moderate & $3497(49.8 \%)$ & $1557(48.6 \%)$ & & $574(45.8 \%)$ & \\
\hline Vigorous & $2055(29.3 \%)$ & $1039(32.4 \%)$ & & $432(34.5 \%)$ & \\
\hline
\end{tabular}

$S D$ standard deviation, $S E P$ socioeconomic position

* $P$ value from $t$ test (age) and Chi-square test (all other variables) comparing wave 2 with wave shown the opposite, however, and such factors (including those not measured in this study) are recognised to cluster at the individual level [17].

The slight decline in this study may reflect a trend at the population level. If so a possible explanation is the increasing prevalence of raised BMI in adults over time [18]. We observed slightly higher mean BMI in the more recent waves, as well as a cross-sectional positive association between BMI and grip strength. We might, therefore, have expected to see greater strength in the more recent waves as opposed to a slight decline. A potential explanation for this discrepancy is the hypothesis that cumulative exposure to raised BMI earlier in the life course might have deleterious effects on grip strength, perhaps due to persistent exposure to a pro-inflammatory state [19]. This idea is supported by the finding that raised BMI across adulthood is associated with reduced upper limb muscle quality in early old age [20].

Another related possibility is that more recent cohorts may have previously undertaken less physical activity, with greater activity across adulthood being shown to have cumulative benefits for grip strength in old age [21]. We saw higher current levels of physical activity in the more recent waves although we could hypothesise that participants in the most recent waves have undertaken less activity across the life course. There are few data for the secular trends in physical activity in adults, however, and those that are available suggest a mixed picture of increasing leisure-time activity contrasted with increased sedentary behaviour and reduced occupational activity [22-24].

The secular trend for three Central European countries within the Survey of Health, Ageing and Retirement in Europe (Germany, The Netherlands and Belgium) over the same period as our study was also found to be slightly negative: a decline of $0.8 \mathrm{~kg}$ on average across age and gender strata between 2004-2005 and 2013 [9]. This contrasts with findings from the same study for Northern (Denmark and Sweden) and Southern (Italy and Spain) European countries where a secular increase in grip strength was seen.

\section{Strengths and limitations}

We used data from a large English cohort which has been considered broadly representative of the underlying population [10]. We also expressed grip strength on the $Z$-score scale using existing British normative data; this allowed us to pool values for use in analyses. Limitations of our study include the fact that there were fewer older participants with their first measurement of grip strength at waves 4 and 6 of the study. There may, therefore, be differences in the trend by age group (or birth cohort) that we lacked the power to 
Fig. 2 Mean grip strength of included participants by gender, wave and age category (unadjusted). $* P<0.05$ and $* * P<0.01$ for comparison of mean grip strength in wave shown compared to equivalent value in wave 2
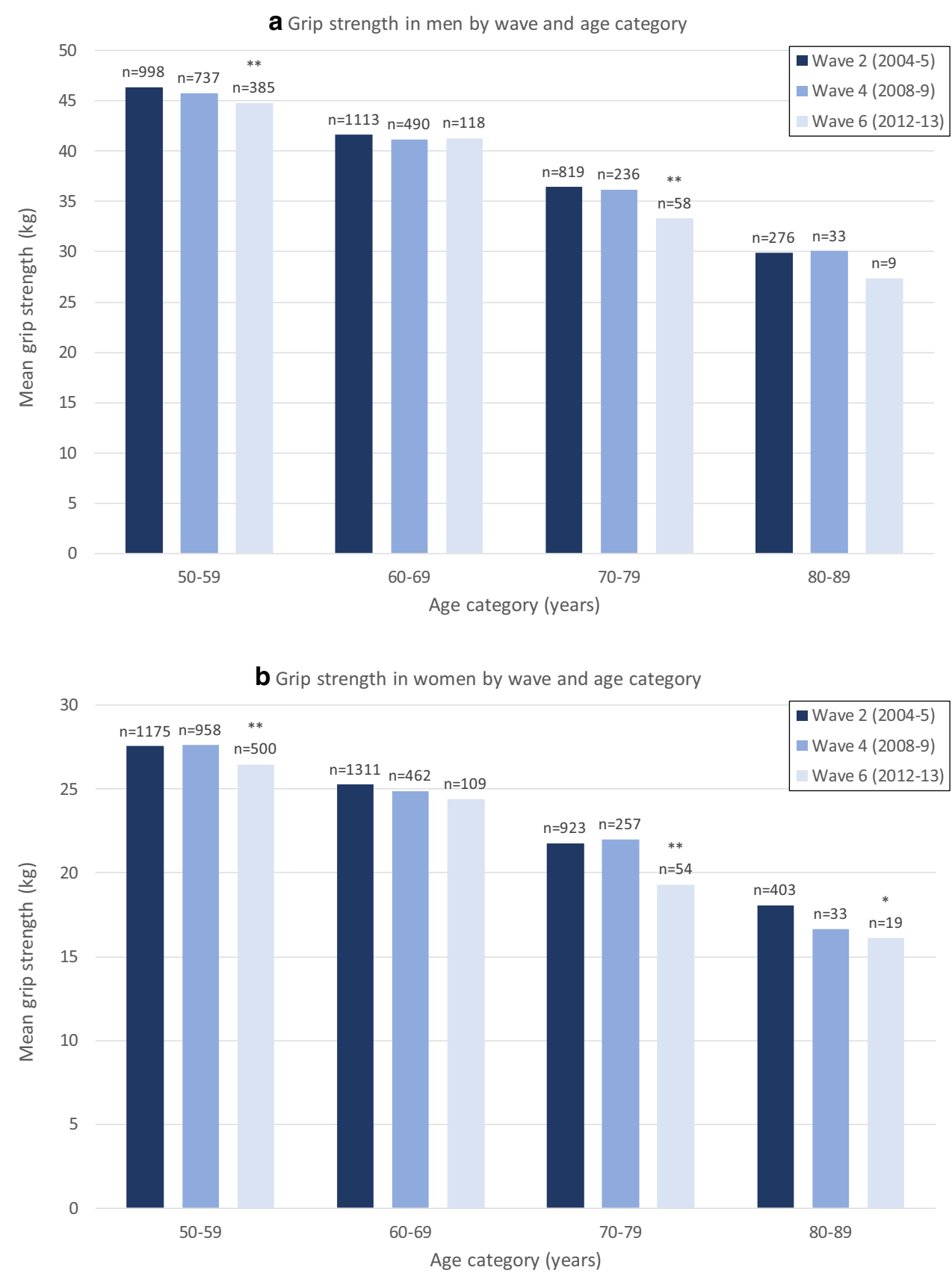

detect. There are also other potential confounders of the secular trend that we did not include such as nutritional status, functional impairments and multimorbidity.

\section{Implications for clinical practice and future research}

Grip strength is of growing relevance to clinical practice, having been recommended as one of two measures used to detect reduced skeletal muscle strength in sarcopenia [5]. Secular trends towards lower mean grip strength would, therefore, be important as they would mean a growing 
Table 2 Results from multivariable linear regression model for grip strength $Z$-score

\begin{tabular}{llll}
\hline Variable & $\beta$ & $(95 \% \mathrm{CI})$ & $P^{*}$ \\
\hline $\begin{array}{l}\text { Years since 2004 } \\
\text { Occupational SEP }\end{array}$ & -0.03 & $(-0.03,-0.02)$ & $<0.01$ \\
$\quad$ Routine & $($ Ref $)$ & & $<0.01$ \\
Intermediate & 0.17 & $(0.12,0.21)$ & \\
Managerial & 0.23 & $(0.19,0.27)$ & $<0.01$ \\
BMI & & & \\
$<20$ & $($ Ref $)$ & & \\
$\geq 20$ and $<25$ & 0.25 & $(0.14,0.36)$ & \\
$\geq 25$ and $<30$ & 0.36 & $(0.25,0.47)$ & \\
$\geq 30$ & 0.42 & $(0.31,0.53)$ & \\
Smoking & & & $<0.01$ \\
Current & $($ Ref $)$ & & \\
Previous & 0.07 & $(0.02,0.12)$ & \\
Never & 0.04 & $(-0.02,0.09)$ & \\
Physical activity & & & \\
Inactive & $($ Ref $)$ & & \\
Mild & 0.12 & $(0.04,0.20)$ & \\
Moderate & 0.4 & $(0.33,0.47)$ & \\
Vigorous & 0.54 & $(0.47,0.62)$ & \\
\hline
\end{tabular}

$N=11,476$. The outcome, grip strength $Z$-score, incorporates participants' age and gender and so these are not included as predictors in the model

SEP socioeconomic position

* $P$ value comparing multivariable models with and without the variable shown

proportion of older people falling below the cut-points for this condition, which has been linked to subsequent falls, fractures, mobility impairment and impairment of activities of daily living. Recent European and US findings suggest differences by country in the secular trend for grip strength and further research to understand these differences is warranted $[9,25]$.

\section{Conclusions}

We found evidence of a small secular decline in mean grip strength in an English ageing cohort between 2004 and 2013, similar to findings in Germany, the Netherlands and Belgium. Further monitoring of secular trends in grip strength and research into the reasons underpinning these regional differences are warranted, given the adverse health outcomes linked to weak grip strength.

Funding AAS is Director of the NIHR Newcastle Biomedical Research Centre. The research was supported by the National Institute for Health Research (NIHR) Newcastle Biomedical Research Centre based at
Newcastle upon Tyne Hospitals NHS Foundation Trust and Newcastle University. The views expressed are those of the author(s) and not necessarily those of the NHS, the NIHR or the Department of Health and Social Care.

\section{Compliance with ethical standards}

Conflict of interest On behalf of all authors, the corresponding author states that there is no conflict of interest.

Ethical approval Ethical approval for all ELSA waves was obtained from NHS Research Ethics Committees under the National Research and Ethics Service.

Informed consent Participants provided informed consent for all aspects of the fieldwork.

OpenAccess This article is distributed under the terms of the Creative Commons Attribution 4.0 International License (http://creativeco mmons.org/licenses/by/4.0/), which permits unrestricted use, distribution, and reproduction in any medium, provided you give appropriate credit to the original author(s) and the source, provide a link to the Creative Commons license, and indicate if changes were made.

\section{References}

1. den Ouden MEM, Schuurmans MJ, Arts IEMA, van der Schouw YT (2011) Physical performance characteristics related to disability in older persons: a systematic review. Maturitas 69(3):208-219

2. Dodds RM, Kuh D, Sayer AA, Cooper R (2018) Can measures of physical performance in mid-life improve the clinical prediction of disability in early old age? Findings from a British birth cohort study. Exp Gerontol 110:118-124

3. Cooper R, Kuh D, Cooper C et al (2011) Objective measures of physical capability and subsequent health: a systematic review. Age Ageing 40(1):14-23

4. Cooper R, Kuh D, Hardy R, Mortality Review Group (2010) Objectively measured physical capability levels and mortality: systematic review and meta-analysis. BMJ 341:c4467

5. Cruz-Jentoft AJ, Bahat G, Bauer J et al (2018) Sarcopenia: revised European consensus on definition and diagnosis. Age Ageing. https://doi.org/10.1093/ageing/afy 169

6. Malina RM (2004) Secular trends in growth, maturation and physical performance: a review. Anthropol Rev 67:3-31

7. Nahhas RW, Choh AC, Lee M et al (2010) Bayesian longitudinal plateau model of adult grip strength. Am J Hum Biol 22(5):648-656

8. Steiber N (2015) Population aging at cross-roads: diverging secular trends in average cognitive functioning and physical health in the older population of Germany. PLoS One 10(8):e0136583

9. Ahrenfeldt LJ, Lindahl-Jacobsen R, Rizzi S, Thinggaard M, Christensen K, Vaupel JW (2018) Comparison of cognitive and physical functioning of Europeans in 2004-2005 and 2013. Int J Epidemiol 47(5):1518-1528

10. Steptoe A, Breeze E, Banks J, Nazroo J (2013) Cohort profile: the English Longitudinal Study of Ageing. Int J Epidemiol 42(6): 1640-1648

11. Banks J, Blake M, Clemens S et al (2018) English Longitudinal Study of Ageing: waves 0-8, 1998-2017, 28th edn. UK Data Service, Colchester, United Kingdom. https://doi.org/10.5255/ukdasn-5050-15 (SN 5050) 
12. Rose D, Pevalin D, O'Reilly K (2005) The national statistics socio-economic classification: origins, development and use. Palgrave Macmillan, Basingstoke

13. Dhalwani NN, O'Donovan G, Zaccardi F et al (2016) Long terms trends of multimorbidity and association with physical activity in older English population. Int J Behav Nutr Phys Act 13(1):1-9

14. Roberts HC, Denison HJ, Martin HJ et al (2011) A review of the measurement of grip strength in clinical and epidemiological studies: towards a standardised approach. Age Ageing 40(4):423-429

15. Dodds RM, Syddall HE, Cooper R et al (2014) Grip strength across the life course: normative data from twelve British studies. PLoS One 9(12):e113637

16. StataCorp (2015) Stata statistical software: release 14. StataCorp, College Station, Texas, USA

17. Robinson SM, Jameson KA, Syddall HE, Dennison EM, Cooper C, Aihie Sayer A (2013) Clustering of lifestyle risk factors and poor physical function in older adults: the Hertfordshire cohort study. J Am Geriatr Soc 61(10):1684-1691

18. Wang YC, Mcpherson K, Marsh T, Gortmaker SL, Brown M (2011) Health and economic burden of the projected obesity trends in the USA and the UK. Lancet 378(9793):815-825

19. Silverman IW (2015) Age as a moderator of the secular trend for grip strength in Canada and the United States. Ann Hum Biol 42(3):201-211

20. Cooper R, Hardy R, Bann D et al (2014) Body mass index from age 15 years onwards and muscle mass, strength, and quality in early old age: findings from the MRC National Survey of Health and Development. J Gerontol A Biol Sci Med Sci 69(10):1253-1259

21. Dodds R, Kuh D, Aihie Sayer A, Cooper R (2013) Physical activity levels across adult life and grip strength in early old age: updating findings from a British birth cohort. Age Ageing 42(6):794-798

22. Stamatakis E, Ekelund U, Wareham NJ (2007) Temporal trends in physical activity in England: the Health Survey for England 1991 to 2004. Prev Med (Baltim) 45(6):416-423

23. Duncan MJ, Vandelanotte C, Caperchione C, Hanley C, Mummery WK (2012) Temporal trends in and relationships between screen time, physical activity, overweight and obesity. BMC Public Health 12(1060):1-9

24. Canizares M, Badley EM (2018) Generational differences in patterns of physical activities over time in the Canadian population: an age-period-cohort analysis. BMC Public Health 18(304):1-11

25. Loprinzi PD (2018) An initial assessment of secular trends in muscular strength among children, adolescents, and adults across the lifespan: national sample of Americans. Am J Heal Promot 32(3):705-707

Publisher's Note Springer Nature remains neutral with regard to jurisdictional claims in published maps and institutional affiliations. 